
\title{
3 Research Square \\ The Multiple Infections of Porcine Diarrhea Viruses in Local Area Based on A Luminex xTAG Multiplex Detection Method
}

Huili liu ( $\sim$ huilil@163.com )

Shanghai Academy of Agricultural Sciences

\section{Ying Shi}

Instite of Animal Science \& Veterinary Medicine『Shanghai Academy of Agricultural Sciences

\section{Benqiang Li}

Institute of Animal Sciences \& Veterinary Medicine, Shanghai Veterinary Research Institute, Shanghai Academy of Agricultural Sciences

Jie Tao

Instite of Animal Science \& Veterinary Medicine

Jinghua Cheng

Institue of Animal Science \& Veterinary Medicine

\section{Research}

Keywords: Porcine viral diarrhea, Luminex xTAG multiplex detection method, Clinical diarrhea sample detection, Multiple pathogen infection

Posted Date: June 10th, 2020

DOl: https://doi.org/10.21203/rs.3.rs-33119/v1

License: (c) (i) This work is licensed under a Creative Commons Attribution 4.0 International License. Read Full License 


\section{Abstract \\ Background}

Porcine viral diarrhea can cause great damage to the pig industry. However, multiple infections have contributed to the poor control of diarrhea, which has also resulted in great difficulties in determining the main pathogenic factors.

\section{Methods}

A Luminex XTAG multiplex detection method was developed for the detection of 11 viral diarrhea pathogens, which allows for the simultaneous qualitative and quantitative detection of viral diarrhea pathogens in clinical samples. A total of 518 porcine stool specimens were collected from 9 pig herds in Shanghai, China from 2015 to 2017, and the pathogen spectrums and co-infections were analyzed.

\section{Results}

The minimum detection rate of the Luminex XTAG multiplex detection method was at least 10 times higher than the traditional PCR method. Of the 518 diarrhea samples, PEDV was found in $17.57 \%$ (91/518), PKoV in 40.35\% (209/518), PAstV in 26.64\% (138/518), PSV in 15.06\% (78/518), PoSaV in $13.13 \%$ (68/518), PTV in 5.21\% (27/518), PDCoV in 4.83\% (25/518), PoRV in 3.28\% (17/518), TGEV in $3.09 \%$ (16/518), PToV in 1.93\% (10/518), and BVDV in 1.74\% (9/518), respectively. Furthermore, multiple infections were commonly seen, with positive rate of $35.14 \%$. Infection pattern of the viral diarrheal pathogens in a specific farm was changing, and different farms had the various diarrhea infection patterns. A longitudinal investigation showed that PEDV was still the key pathogen which was closely related to the death of diarrhea piglets. Other pathogens might play synergistic roles in the pathogenesis of diarrhea disease.

\section{Conclusions}

Here we provided a Luminex xTAG multiple detection method for viral diarrhea pathogen infection in clinical, which was more sensitive and specific than general multiplex PCR method. Furthermore, the surveillance confirmed high infection rate of PKoV, but PEDV was still the key pathogen and multiple pathogens synergistically complicated the infection status in southern China, suggesting that controlling porcine diarrhea might be more complex than previously thought. A better understanding of viruses that cause diarrhea in piglets will aid in better preventing and controlling epidemics of viral porcine diarrhea.

\section{Introduction}


In recent years, viral diarrhea in pig herds has led to serious problems with clinical symptoms of diarrhea, vomiting and dehydration, thereby affecting pig growth and leading to huge economic loss [1]. This disease increases the infection rate to piglets and mortality, which could reach up to $100 \%$ [2].

Furthermore, multiplex infection and synergistic infection, which are commonly observed in clinic, pose a new challenge to disease diagnosis and control. The viruses that cause porcine diarrhea disease are diverse, including porcine epidemic diarrhea virus (PEDV) [3-5], porcine deltacorona virus (PDCoV), porcine transmissible gastroenteritis virus (TGEV) [6], porcine teschvirus (PTV), bovine viral diarrhea virus (BVDV), porcine rotavirus (PoRV), porcine sapelovirus (PSV), porcine kobuvirus (PKoV), porcine astrovirus (PAstV), porcine torovirus (PToV), porcine sapovirus (PoSaV), etc. An infection with any of these viruses can develop into similar clinical symptoms, including severe diarrhea and dehydration, and in some cases, multiplex infections of these pathogens are common. However, it remains difficult to distinguish these pathogens in clinic [4, 16-18].

Although regular vaccine immunization has been strictly conducted, the high morbidity of diarrhea remains as a serious problem, which needs to be solved in time. Therefore, the epidemiology of diarrhea viruses needs to be investigated, in order to identify the dominant viruses. Thus, the surveillance of porcine is warranted to better understand the evolution in the field. Laboratory detection methods include virus isolation, enzyme linked immunosorbent assay (ELISA) and polymerase chain reaction (PCR). However, virus isolation needs long period and complicated operations, and ELISA is mainly used for epidemiological survey. Therefore, it is important and urgent to establish a detection method with high specificity and high sensitivity, which can meet the needs of multiple pathogen detection at the same time. As the multiplex reverse transcription-polymerase chain reaction (RT-PCR) method has high sensitivity, specificity and could simultaneously detect a variety of pathogen, it is more suitable for rapid diagnosis of multiplex infection. Therefore, in this study, a multiple detection method for viral diarrhea was developed.

The precise data about the prevalence of multiple infections in porcine and wild boars have only been reported in a limited number of countries $[10,11]$. Moreover, the prevalence and multiplex infections of porcine diarrhea in Shanghai have never been studied. Therefore, the epidemiology is needed to determine the prevalence in these circulating strains, in order to develop vaccination programs and establish a surveillance system.

Co-infection with various viruses makes preventing and curing diarrhea in pigs more complex. Therefore, we performed an epidemiological study to identify the frequency of these viruses in cases of porcine viral diarrhea in Southern China. Furthermore, the multiplex infection situation was analyzed, which will pave the way to improving the strategies in preventing and controlling virus infection in swine farms.

\section{Materials And Methods}

\section{Specimens}


A total of 518 porcine stool specimens were collected from 9 pig herds in Shanghai, China from 2015 to 2017. Pigs of all ages were sampled and 1 to 3 -week-old piglets were particularly collected. Antibiotic treatment was invalid for all sampled pigs. Each sample was suspended in phosphate-buffered saline (PBS) containing $1,000 \mathrm{U} / \mathrm{ml}$ penicillin and $1,000 \mathrm{U} / \mathrm{ml}$ streptomycin and centrifuged at 12,000 r.p.m. for 10 minutes. A portion of the suspension was used for RNA extraction, while the remaining supernatants were stored at $-70^{\circ} \mathrm{C}$.

\section{Establishment of Luminex xTAG multiplex detection method}

According to the conserved sequences in GenBank, DNAStar and Oligo7 software were used to design the PCR primer pairs of 11 pathogens (Table 1). The $\mathrm{M}$ gene of PToV, $\mathrm{M}$ gene of PDCoV, RDRP gene of PAstV, 3D gene of PKoV, RDRP gene of PSV, 3D gene of PTV, RDRP gene of PsaV, 5' URT gene of BVDV, M gene of PEDV, N gene of TGEV and VP6 gene of PoRV were as target genes. Spacer C12 was added between the $5^{\prime}$ end of all upstream primers and the $3^{\prime}$ end of the anti-TAG sequence, and the $5^{\prime}$ end of all downstream primers was biotinylated (Biotin-) tag; selecting TAG microspheres complementary to antiTAG sequence; hybridizing biotinylated tagged amplification product with TAG microspheres, and obtaining the hybridization product for liquid-phase chip detection, and detecting the pathogen according to the MFI value. The specificity and sensitivity of Luminex XTAG assay method were analyzed. And the hybridization system and reaction conditions were also optimized. 
Table 1

The PCR primer pairs of 11 pathogens. The $M$ gene of PToV, $M$ gene of PDCoV, RDRP gene of PAstV, 3D gene of PKoV, RDRP gene of PSV, 3D gene of PTV, RDRP gene of PsaV, 5 ' URT gene of BVDV, $\mathrm{M}$ gene of PEDV, $\mathrm{N}$ gene of TGEV and VP6 gene of PoRV were chosen as target genes. Spacer C12 was added between the $5^{\prime}$ end of all upstream primers and the $3^{\prime}$ end of the anti-

TAG sequence, and the $5^{\prime}$ end of all downstream primers was biotinylated (Biotin-) tag, selecting TAG microspheres complementary to anti-TAG sequence.

\begin{tabular}{|c|c|c|}
\hline Virus & Gene & sequence $\left(5^{\prime}-3^{\prime}\right)$ \\
\hline \multirow[t]{2}{*}{ PToV } & \multirow[t]{2}{*}{ M } & ААСTTTСTСТСТСТАTTCTTATTT/iSpC12/AATTGCTTATTGGTGGCTTC \\
\hline & & Biotin-AGCDATTTGRGCDGCATTC \\
\hline \multirow[t]{2}{*}{ PDCoV } & \multirow[t]{2}{*}{ M } & СTATCATTTATCTCTTTCTCAATT/ISpC12/CGCAGTTTTCATTGTGTCCA \\
\hline & & Biotin-CCTGTGGCGGATTTCTAACT \\
\hline \multirow[t]{2}{*}{ PAstV } & \multirow[t]{2}{*}{ RDRP } & САATTTACATTTCACTTTCTTATC/iSpC12/CCTTWCCCCACTGATGAAGA \\
\hline & & Biotin-CCTGTCCATCTGCCTTTCTGT \\
\hline \multirow[t]{2}{*}{ PKoV } & \multirow[t]{2}{*}{ 3D } & TCATCACTTTCTTTACTTTACATT/iSpC12/CGCCGTTCACTCTTTGTCC \\
\hline & & Biotin-ACCAAGCAGCATCCTACCAG \\
\hline \multirow[t]{2}{*}{ PSV } & \multirow[t]{2}{*}{ RDRP } & АATTTCTTCTCTTTCTTTCACAAT/iSpC12/CTGGACTGGGCCTATACT \\
\hline & & Biotin-TYAGGTACACACGGGCTC \\
\hline \multirow[t]{2}{*}{ PTV } & \multirow[t]{2}{*}{$3 \mathrm{D}$} & ACACTCATTTAACACTATTTCATT/ISpC12/TGCAGCTCTTTCACATTTRGA \\
\hline & & Biotin-ACTTGTATGAGGCCCATCG \\
\hline \multirow[t]{2}{*}{ PoSaV } & \multirow[t]{2}{*}{ RDRP } & ACAAATATCTAACTACTATCACAA/iSpC12/GTGGCAACGTACAAYGCRTGG \\
\hline & & Biotin-GCCTCCATCACGAACACT \\
\hline \multirow[t]{2}{*}{ BVDV } & \multirow[t]{2}{*}{ 5’URT } & ATACTTTACAAACAAATAACACAC/iSpC12/AGCCATGCCCHTAGTAGGAC \\
\hline & & Biotin-CCTCTGCWRCACCCTATC \\
\hline \multirow[t]{2}{*}{ PEDV } & \multirow[t]{2}{*}{ M } & СТTTCTCATACTTTCAACTAATTT/iSpC12/GTCAAGATGGCTATTCTATGG \\
\hline & & Biotin-ACCAAGAATGTGTCCTGCG \\
\hline \multirow[t]{2}{*}{ TGEV } & \multirow[t]{2}{*}{$\mathrm{N}$} & TTAACAACTTATACAAACACAAAC/iSpC12/CAAAGGAATAGGTAACAGGG \\
\hline & & Biotin-ATCTGCATGAGGTCCAGT \\
\hline \multirow[t]{2}{*}{ PoRV } & \multirow[t]{2}{*}{ VP6 } & CATAATCAATTTCAACTTTCTACT/iSpC12/GGATTACTTGGYACTACTTT \\
\hline & & Biotin-AATGATGCTGAYTATGGAAGT \\
\hline
\end{tabular}


According to manufacturer's instructions, total RNA was extracted from the fecal supernatant using TRIzol reagent (Life Technologies, Gaithersburg, MD, USA). Viral RNA was converted to cDNA using reverse transcriptase M-MLV (TaKaRa, Dalian, China) in a final volume of $20 \mu \mathrm{L}$ containing $5 \mu \mathrm{L}$ RNA, 4 $\mu \mathrm{L} 5 \times \mathrm{RT}$ buffer, $2 \mu \mathrm{L}$ dNTPs $(10 \mathrm{mM}), 2 \mu \mathrm{L} 10 \mu \mathrm{M}$ random primer, $0.5 \mu \mathrm{L}$ MLV reverse transcriptase, 0.5 $\mu \mathrm{L}$ RNase inhibitor (TaKaRa, Dalian, China), and $6 \mu \mathrm{L}$ diethylpyrocarbonate (DEPC)-treated water. The reaction was incubated at $42^{\circ} \mathrm{C}$ for 1 hour, followed by incubation at $70{ }^{\circ} \mathrm{C}$ for 15 minutes. Afterwards, the cDNA was screened using the method we had established.

\section{Longitudinal investigation of enteric pathogens}

A longitudinal follow-up study was setup between February and April in 2017. To warrant the health status of the pig stock, entrance to the farm was strictly regulated, and sampling was performed by the farmer. Detailed instructions and sampling materials were provided to the farmer. A dry cotton rectal swab was collected from each individual pig with diarrhea and was placed immediately in $2 \mathrm{ml}$ of viral transport medium (phosphate buffered saline containing 1,000 U/ml penicillin and 1,000 U/ml streptomycin) and stored at $-20^{\circ} \mathrm{C}$. The farmer was asked to mark the tube of each sample for diarrheic signs. Every week, samples were collected from the farm and transported to the laboratory for pathogen detection.

\section{Results}

\section{Establishment of Luminex xTAG multiplex detection method}

Based on a single detection system, a Luminex XTAG multiplex detection method for the simultaneous detection of 11 diarrheal viruses was established. The optimal hybridization system and reaction condition were as follows: $20 \mu \mathrm{L}$ microsphere working solution, $5 \mu \mathrm{L}$ PCR amplification product, and $75 \mu \mathrm{L}$ SAPE report buffer; the results of optimal hybridization temperature was $42^{\circ} \mathrm{C}$, and the best hybridization time was $30 \mathrm{~min}$. The specificity of Luminex XTAG assay method was tested, which showed that each primer pair had good specificity and there was no cross-reaction between the primer pairs (Figure 1A). The sensitivity test of the Luminex XTAG detection method (Figure 1B) showed that the minimum detection limit was PTV in $3.12 \times 10^{3}$ copies/ $\mu \mathrm{L}$, PKOV in $2.92 \times 10^{3}$ copies $/ \mu \mathrm{L}, \mathrm{PDCoV}$ in $2.79 \times 10^{3}$ copies/ $\mu \mathrm{L}, \mathrm{PSV}$ in $3.37 \times 10^{3}$ copies $/ \mu \mathrm{L}, \mathrm{PoSaV}$ in $2.7 \times 10^{3}$ copies $/ \mu \mathrm{L}, \mathrm{PAst} V$ in $3.02 \times 10^{3}$ copies $/ \mu \mathrm{L}, \mathrm{PToV}$ in $2.65 \times 10^{3}$ copies/ $\mu \mathrm{L}, \mathrm{PoRV}$ in $2.57 \times 10^{3}$ copies/ $\mu \mathrm{L}$, PEDV in $1.74 \times 10^{3}$ copies $/ \mu \mathrm{L}, \mathrm{BVDV}$ in $2.41 \times 10^{3}$ copies $/ \mu \mathrm{L}$, and TGEV in $2.75 \times 10^{3}$ copies/ $\mu \mathrm{L}$, respectively. The minimum detection rate was at least 10 times higher than the traditional multiplex PCR method.

The pathogen composition in each sample could be estimated intuitively. The concentration of different pathogens in each sample was presented as different colors in a heat-map (Figure 2). Therefore, Different colors represent different concentrations of pathogens. The closer the color was to black, the lower concentration was present; and the closer the color was to red, the higher concentration was present in the sample. In addition $\nabla$ the predominant viruses in the multiplex infection samples could be speculated according to quantitative analysis. Therefore, in virtue of the quantitative analysis, the detection results will be more clearly visible and targeted prevention or therapy may be carried out in the pigsty, paving the way to instructing the clinical production. 


\section{Viral pathogens infected in diarrhea stools were diversified}

A total of 518 porcine stool specimens from five districts in Shanghai were detected using multiplex RTPCR method. Of the 518 diarrhea samples, PEDV was found in $17.57 \%$ (91/518), PKoV in $40.35 \%$ (209/518), PAstV in 26.64\% (138/518), PSV in $15.06 \%$ (78/518), PoSaV in 13.13\% (68/518)区PTV in $5.21 \%(27 / 518) \bowtie P D C o V$ in $4.83 \%$ (25/518), PoRV in 3.28\% (17/518), TGEV in 3.09\% (16/518), PToV in $1.93 \%$ (10/518), BVDV in 1.74\% (9/518), respectively (Table 2 \& Figure 3$)$. Therefore, complicated pathogen composition existed in clinic, which emphasized the importance of monitoring the fluctuant infection spectrum to guide the clinical production.

\begin{tabular}{ccc}
\hline Virus & Positive numbers & Positive percent \\
\hline PKOV & 209 & $40.35 \%$ \\
\hline PASTV & 138 & $26.64 \%$ \\
\hline PEDV & 91 & $17.57 \%$ \\
\hline PSV & 78 & $15.06 \%$ \\
\hline POSAV & 68 & $13.13 \%$ \\
PTV & 27 & $5.21 \%$ \\
\hline PDCOV & 25 & $4.83 \%$ \\
\hline PORV & 17 & $3.28 \%$ \\
\hline TGEV & 16 & $3.09 \%$ \\
\hline PTOV & 10 & $1.93 \%$ \\
\hline BVDV & 9 & $1.74 \%$ \\
\hline
\end{tabular}

Table 2. Identification of various porcine diarrhea viruses. A total of 518 porcine stool specimens from five districts in Shanghai were detected by multiplex RT-PCR method.

\section{Multiple infections were commonly seen in clinic}

A total of 182 co-infections were identified, with positive rate of $35.14 \%$. Among the 11 enteric viruses, $50 \%(91 / 182)$ of samples had at least two pathogens, and $32.97 \%(60 / 182)$ of samples had three different pathogens, while the positive rate of quadruple- and quintuple-infection was $13.19 \%$ and $2.20 \%$, respectively. Most surprisingly was that six (1.65\%) viral diarrhea pathogens were simultaneously detected from one diarrhea stool, indicating a complex diarrhea pathogen infection pattern and pathogenesis in clinic (Figure 4A \& Table 3).

\begin{tabular}{ccc}
\hline Complex infection & Positive numbers & Positive percent \\
\hline Dual- & 91 & $50.00 \%$ \\
Triple- & 60 & $32.97 \%$ \\
Quadruple- & 24 & $13.19 \%$ \\
Quintuple- & 4 & $2.20 \%$ \\
Sextuple- & 3 & $1.65 \%$
\end{tabular}

Table 3. The co-infections of all clinical samples. A total of 182 co-infections were identified, with positive rate of $35.14 \%$. Different multiplex infections had been shown in the table. 
Among the samples positive for the 11 enteric viruses, PKoV exhibited high co-infection rates, ranging from $2.20 \%$ to $32.97 \%$ (Figure 4B). Of the 91 dual-infections, 56 samples were PKoV-positive (61.54\%) and 17 samples were PEDV-positive (18.68\%). The co-infection of PKoV-positive samples with PAstV, PoSaV, and PEDV reached 32.97\%, 10.99\% and 4.40\%, respectively (Figure 4B). Of the 91 PEDV-positive samples, dual-infection with PKoV, PSV and PAstV reached $4.4 \%, 7.69 \%$ and $6.59 \%$, respectively (Figure 4B). Of the 60 triple-infections, 39 samples were PKoV-positive (65\%) and 22 samples were PEDV-positive (37.75\%), while 10 (16.67\%) samples were co-infected with PKOV, PEDV and other diarrhea viruses (Figure 4C). Among the 24 quadruple-infections, 11 samples were PKoV-positive (45.83\%) and 6 samples were PEDV-positive (25\%). All the quintuple and sextuple-infections were positive with PKoV and PEDV. There were no cases of co-infection with any combination of PEDV, TGEV, and PRoV. Interestingly, coinfections with PEDV and PKoV accounted for 31.87\% (29/91) of the PEDV positive samples. PKoV was always one of the co-infecting viruses, which indicated that PKoV might play synergistic roles in the pathogenesis of diarrhea disease.

\section{Different farms showed variable infection spectrum}

The composition of enteric pathogens among different farms was analyzed (Figure 5\& Figure 6). In farm A, eleven kinds of pathogens were detected including multiple co-infections (dual-, triple-, quadruplequintuple- and sextuple-infection). In farm B, only eight kinds of pathogens were identified including dualand triple-infection. It was concluded that the pathogen composition of a specific farm was unique. Therefore, different prevention and control measures should be carried out based on the monitored pathogens.

\section{Viral infection spectrum in a specific farm}

In tracking the annual viral diarrhea tests in one farm from 2015 to 2017, it was observed that the prevalence of the viral diarrhea pathogens also changed over time (Figure 7). In 2015, PEDV had the highest positive rate of $45.83 \%$, while the second highest was PKoV (33.33\%). Accordingly, high diarrhea rate $(52 \%)$ and mortality (7.1\%) were observed. In 2016, PKoV became the most popular pathogen with a particularly high positive rate of $68.67 \%$, and this was closely followed by PAstV (32\%) and PSV (24\%). The PEDV positive rate was merely $12 \%$, accordingly, diarrhea rate decreased to $38 \%$ and mortality to $5.5 \%$. In 2017, PAstV had the highest detection rate of pathogens (52.50\%), followed by PKoV (40\%) and PEDV (17.5\%), while the diarrhea rate and mortality became $42 \%$ and $6.8 \%$, respectively. Taken together, these results do indicate that the fluctuations of diarrhea rate and mortality were accordance with the tendency of PEDV positive rate, while high rate of PKoV or PAstV did not necessarily correspond to high diarrhea rate and mortality.

\section{Longitudinal investigation showed dominant infection agents}

A longitudinal follow-up study was setup between February and April in 2017 to identify the dominant pathogen. A total of 173 diarrhea stools were collected and detected. As a result, 11 viral diarrhea pathogens were detected, and the positive detection rate of PEDV, PKoV, PoRV, PSV, BVDV, PoSaV, PToV, 
PAstV, TGEV, PDCoV, and PTV was 32.37\%, 26.01\%, 14.45\%, 6.94\%, 6.36\%, 11.56\%, 2.89\%, 26.01\%, 3.47\%, $0.58 \%$, and $1.73 \%$, respectively (Table 4 ). A total of 60 co-infections were identified, with positive rate of $34.68 \%$ (60/173) (Table 5). Among the 11 enteric viruses, 48.33\% (29/60) of samples had at least two pathogens, and $31.67 \%$ (19/60) of samples had three different pathogens, while the positive rate of quadruple- and quintuple-infection was $10.00 \%(6 / 60)$ and 5.00\%(3/60), respectively. Six viral diarrhea pathogens were simultaneously detected from one diarrhea stool (5.00\%, 3/60). Of the 56 PEDV-positive samples, co-infection with PKoV and PoRV reached 16.07\% (9/56) and 5.36\% (3/56), respectively. PKoV was always one of the co-infecting viruses; however, the viral load was not extremely high according to the quantitative analysis.

\begin{tabular}{|c|c|c|c|c|c|c|c|c|c|c|c|}
\hline Virus & PEDV & $\mathrm{PKoV}$ & PAstV & PoRV & PoSaV & PSV & BVDV & TGEV & PToV & PTV & PDCoV \\
\hline Positive numbers & 56 & 45 & 45 & 25 & 20 & 12 & 11 & 6 & 5 & 3 & 1 \\
\hline Positive percent & $32.37 \%$ & $26.01 \%$ & $26.01 \%$ & $14.45 \%$ & $611.56 \%$ & $6.94 \%$ & $6.36 \%$ & $3.47 \%$ & $2.89 \%$ & $1.73 \%$ & $0.58 \%$ \\
\hline
\end{tabular}

Table 4. The detection of various pathogens by Luminex xTAG multiplex detection method. 173 diarrhea samples collected in 2017 were further quantitatively detected using the Luminex xTAG multiplex detection method.

\begin{tabular}{ccc}
\hline Complex infection & Positive NO. & Positive percent \\
\hline Dual- & 29 & $16.76 \%$ \\
\hline Triple- & 19 & $10.98 \%$ \\
\hline Quadruple- & 6 & $3.47 \%$ \\
\hline Quintuple- & 3 & $1.73 \%$ \\
\hline Sextuple- & 3 & $1.73 \%$
\end{tabular}

Table 5. The co-infections of clinical samples from a longitudinal follow-up study in 2017. A total of 173 samples were detected. Different multiplex infections had been shown in the table.

The morbidity and mortality rate of the farm during sampling was 57\% and 5.23\%; even vaccinated suckling piglets were not spared. The infected piglets predominantly showed yellow watery stools, weight loss, and death from dehydration. Interestingly, single PEDV infection or co-infection of PEDV with other diarrhea viruses was identified in all the dead piglets. One piglet infected with 6 kinds of diarrhea viruses including PEDV, finally died. While another piglet infected with six other viruses was still alive with great weight loss. The same situation also applied to other multiple infections. All in all, PEDV is still the key pathogen which was closely related to death of diarrhea piglets. Other pathogens might play synergistic roles in the pathogenesis of diarrhea disease. 
According to the present surveillance results, relative proposals and solutions were put forward and carried out to control the emerging diarrhea and to prevent later potential occurrence.

\section{Discussion}

Porcine viral diarrhea disease seriously endangers the development of the pig industry, and leads to significant economic losses for pig farmers worldwide [7]. Clinically, the complexity of the disease has increased. In some cases, multiplex infections with two or more viruses are common, which seriously interfere with the clinical diagnosis [8-12]. It has been speculated that the incidence of diarrhea would decline due to the vaccine prevention of PEDV, TGEV and PoRV triplets. However, diarrhea continued to threaten pig farms. Beside these three traditional porcine viral diarrhea pathogens (PEDV, TGEV and PoRV), other viral diarrhea pathogens have also been reported in recent years [13-20]. In particular, the situation of multiplex infections has become more serious, resulting in increased pressure in the prevention and control of porcine diarrhea. Although the correlation between emerging viruses and diarrhea has not been clearly discussed, these co-infections have indeed enhanced the severity of diarrhea in the present study. Therefore, in order to accurately differentiate the infections in clinical specimens and prevent the transboundary spread of porcine viral diarrhea disease, it is necessary to conduct pathogen monitoring in clinical production.

Currently, PCR-based methods have been proven to be convenient and highly sensitive for detecting porcine diarrhea-associated viruses [2, 21]. The multiple PCR method for testing 4 or 7 kinds of diarrhea pathogens was established in the laboratory of the investigators, and was applied for clinical detection $[21,39]$. However, complicated multiplex infections require a more accurate detection method. Therefore, we further developed Luminex XTAG high-throughput detection method for viral diarrhea pathogens in pigs, which has the advantages of high flux, wide range of detection, and small sample size. Furthermore, this is suitable for the large-scale screening of clinical samples, and is especially suitable for the multiplex infection detection of samples. Using Luminex XTAG technology, the standard curves for the above 11 diarrheal pathogens were established, and the content of each pathogen was calculated by measuring the MFI value of each sample. By analogy, the viral load can be determined for each sample, and finally, the pathogen with the highest risk of infection in each pig farm was analyzed, which would be helpful to guide the formulation of immunity and control measures in pig farms. In addition, this can also intuitively identify the maximum level of pathogens in each sample based on the cluster analysis software.

PEDV has become the most important intestinal pathogen in swine in China [22]. Many studies of the mechanism of PEDV infection and effective vaccines have been published. However, the variations of the virus and its co-infections with other enteric viruses, had contributed to the poor control of PEDV infection. In order to better understand the prevalence of the co-infection in southern China, an epidemiological investigation of porcine diarrhea viruses was carried out in this study. It was reported that the co-infection of PEDV and PBoV was more prevalent in diarrhea samples than non-diarrhea samples [23]. A recent study showed that $27 \%$ of samples had PEDV infection alone, whereas the 
remaining $73 \%$ of samples exhibited two to nine pathogens [24]. According to this survey, single infection with PEDV occurred in only $6.56 \%$ of samples (34 out of 518 ), while most of the samples involved coinfections.

Except for the co-infections with PEDV, various other types of co-infections existed in the study. It was considered that animals co-infected with more than one enteric virus experienced increased intestinal epithelium damage and/or viral replication, which results in more severe diarrhea [10]. Forty samples of diarrhea in piglets in Sichuan Province were tested and five samples (12.5\%) of multiplex infections of PKoV, PAstV and PToV were identified [12]. Chang Tiecheng et al. [13] tested 165 samples obtained from 42 pig farms, and reported that 2 of 42 pig farms were infected with PEDV and TGEV, accounting for $4.76 \%$. Furthermore, seven pig farms were infected with PEDV and PoRV, accounting for $16.67 \%$, and two pig farms were infected with three viruses, accounting for $4.76 \%$. This was consistent with the present results, in which there were serious multiplex infections in these pathogens. Among the 11 enteric viruses, $50 \%(91 / 182)$ of samples had at least two pathogens, and $32.97 \%(60 / 182)$ of samples had three different pathogens, while the positive rate of quadruple- and quintuple-infection was $13.19 \%$ and $2.20 \%$, respectively. Notably, co-infection with six viral diarrhea pathogens was identified in three samples (1.65\%). However, no new vaccines for diarrhea pathogens had been developed and applied to pig farms. Furthermore, there have been instances of co-infections in sows, even though these are usually asymptomatic. This may explain the persistence of viruses within the herd, and facilitation of vertical transmission.

PKoV can infect pigs of all ages and varieties with prevalence of ranges from 19.3-99.0\% in different countries [16]. Since the first report of PKoV in Hungary [25]and China [26], it has been confirmed that PKoV was widely present in several countries, and plays an important role in diarrhea outbreak in pigs [27-31]. The statistical analysis of the PKoV positive rate between diarrheic and healthy pigs, as well as a survey for other enteric pathogens in diarrheic pigs, suggested that PKoV may play a role as a causative agent of gastroenteritis in pigs[28]. Recent studies have revealed the genetic diversity and possible pathogenic role of PKoV in conjunction with other pathogens in piglets $[13,28]$. PKoV has also been linked to porcine diarrhea although its pathogenesis remains unclear (Barry et al., 2011; Chen et al., 2013a). It was reported that piglets with diarrhea shed more Kobuvirus than healthy individuals during the late-nursing stage (6-21 days old) [32], which accounted for the high positive rate of PKoV. Based on the rates of infection documented in this study, PKoV exhibited the highest infection rate (40.35\%), and high co-infection rates ranging from $2.20-32.97 \%$, suggesting a high prevalence of co-infections in the sampled regions. Since PKoV has the highest infection rate and co-infection rate, further investigations should be conducted to research its characteristics and pathogenic mechanism. Interestingly, coinfections with PEDV and PKoV accounted for 31.87\% (29/91) of the PEDV positive samples, which was similar with the frequency of infection with PEDV alone $(37.36 \%, 34 / 91)$. These data suggested that PKoV had a potential role in PEDV-induced diarrhea symptoms, which was consistent with the previous study [32]. The high prevalence of co-infection, particularly PKoV and PEDV, is a cause for concern and should be seriously considered. 
The pathogen composition of different farms varied. Therefore, clinical control should be based on pathogen monitoring. Although infection was frequent in the winter season on farms in the temperate climate, diarrhea infection occurred throughout the year in porcine farms. Furthermore, the pathogen composition was more complex, and multiple infections more frequently emerged. Therefore, seasonal prevention measures are more important to control the diarrhea. The etiology of porcine diarrhea is complex. This might be influenced by bacteria, in addition to viral factors, and the interaction between bacteria and viruses might also contribute to the complexity of the disease. A single pathogen might not be the main cause of diarrhea; however, the unique relationship among different pathogens needs further research. The specific mechanisms of the diarrhea pathogens for porcine diarrhea diseases remain unknown. However, the potential hazards cannot be ignored, and these should be detected and prevented at an early stage.

\section{Conclusions}

In summary, here we provided a Luminex xTAG multiple detection method for viral diarrhea pathogen infection in clinical, which was more sensitive and specific than general multiplex PCR method.

Furthermore, the surveillance confirmed high infection rate of PKoV, but PEDV was still the key pathogen and multiple pathogens synergistically complicated the infection status in southern China, suggesting that controlling porcine diarrhea might be more complex than previously thought. A better understanding of viruses that cause diarrhea in piglets will aid in better preventing and controlling epidemics of viral porcine diarrhea. Furthermore, attention should be given to the relevant relevant vaccines, and the expansion of the vaccine reserve, in order to prepare for the outbreak of a new round of porcine diarrhea disease in advance.

\section{List Of Abbreviations}

Porcine kobuvirus (PKoV), porcine astrovirus (PAstV), porcine epidemic diarrhea virus (PEDV), porcine sapelovirus (PSV), porcine sapovirus (PoSaV), porcine teschovirus (PTV), porcine deltacoronavirus (PDCoV), porcine rotavirus (PoRV), transmissible gastroenteritis virus (TGEV), porcine torovirus (PToV), bovine viral diarrhea virus (BVDV), phosphate-buffered saline (PBS), diethylpyrocarbonate (DEPC), polymerase chain reaction (PCR), enzyme linked immunosorbent assay (ELISA), reverse transcriptionpolymerase chain reaction (RT-PCR).

\section{Declarations}

\section{Ethics approval and consent to participate}

Not applicable.

\section{Consent for publication}

Not applicable. 
Availability of data and materials

All data generated or analyzed during this study are included in this published article [and its supplementary information files.

\section{Competing interests}

The authors declare no competing financial interests.

\section{Funding}

This work was supported by the National Key Research and Development Program of China (Grant No. 2018YFD0500102) and the Shanghai Agriculture Applied Technology Development Program, China (No. 2019-3-2).

\section{Author Contributions}

H.L. designed the research, Y.S., B.L, J.T., and J.CH. performed research; Y. S., B.L. and H.L. analyzed data; Y.S., B.L., and H.L. wrote the paper.

\section{Acknowledgments}

Not applicable.

\section{References}

1. Zhao J, Shi BJ, Huang XG, Peng MY, Zhang XM, He DN, Pang R, Zhou B, Chen PY. A multiplex RT-PCR assay for rapid and differential diagnosis of four porcine diarrhea associated viruses in field samples from pig farms in East China from 2010 to 2012. J Virol Methods. 2013;194:107-12.

2. Jia S, Feng B, Wang Z, Ma Y, Gao X, Jiang Y, Cui W, Qiao X, Tang L, Li Y, et al. Dual priming oligonucleotide (DPO)-based real-time RT-PCR assay for accurate differentiation of four major viruses causing porcine viral diarrhea. Mol Cell Probes. 2019;47:101435.

3. Pensaert MB, de Bouck P. A new coronavirus-like particle associated with diarrhea in swine. Arch Virol. 1978;58:243-7.

4. Stevenson GW, Hoang H, Schwartz KJ, Burrough ER, Sun D, Madson D, Cooper VL, Pillatzki A, Gauger $\mathrm{P}$, Schmitt BJ, et al. Emergence of Porcine epidemic diarrhea virus in the United States: clinical signs, lesions, and viral genomic sequences. J Vet Diagn Invest. 2013;25:649-54.

5. Wood EN. An apparently new syndrome of porcine epidemic diarrhoea. Vet Rec. 1977;100:243-4.

6. Doyle LP, Hutchings LM. A transmissible gastroenteritis in pigs. J Am Vet Med Assoc. 1946;108:2579.

7. Chuchaona W, Khamrin P, Yodmeeklin A, Kongkaew A, Vachirachewin R, Kumthip K, Ushijima H, Maneekarn N. Detection and molecular characterization of porcine kobuvirus in piglets in 2009- 
2013 in northern Thailand. Trop Anim Health Prod. 2017;49:1077-80.

8. Niederwerder MC, Hesse RA. Swine enteric coronavirus disease: A review of 4 years with porcine epidemic diarrhoea virus and porcine deltacoronavirus in the United States and Canada. Transbound Emerg Dis. 2018;65:660-75.

9. Jung K, Hu H, Eyerly B, Lu Z, Chepngeno J, Saif LJ. Pathogenicity of 2 porcine deltacoronavirus strains in gnotobiotic pigs. Emerg Infect Dis. 2015;21:650-4.

10. Tuanthap S, Phupolphan C, Luengyosluechakul S, Duang-In A, Theamboonlers A, Wattanaphansak S, Vongpunsawad S, Amonsin A, Poovorawan Y. Porcine rotavirus $C$ in pigs with gastroenteritis on Thai swine farms, 2011-2016. PeerJ. 2018;6:e4724.

11. Amimo JO, Vlasova AN, Saif LJ. Detection and genetic diversity of porcine group A rotaviruses in historic (2004) and recent (2011 and 2012) swine fecal samples in Ohio: predominance of the G9P[13] genotype in nursing piglets. J Clin Microbiol. 2013;51:1142-51.

12. Chen Q, Gauger P, Stafne M, Thomas J, Arruda P, Burrough E, Madson D, Brodie J, Magstadt D, Derscheid $\mathrm{R}$, et al. Pathogenicity and pathogenesis of a United States porcine deltacoronavirus cell culture isolate in 5-day-old neonatal piglets. Virology. 2015;482:51-9.

13. Zhai SL, Zhang H, Lin T, Chen SN, Zhou X, Chen QL, Lv DH, Wen XH, Zhou XR, Jia CL, Wei WK. A novel porcine kobuvirus emerged in piglets with severe diarrhoea in China. Transbound Emerg Dis. 2017;64:1030-6.

14. Tao J, Liao J, Wang Y, Zhang X, Wang J, Zhu G. Bovine viral diarrhea virus (BVDV) infections in pigs. Vet Microbiol. 2013;165:185-9.

15. Prodelalova J. The survey of porcine teschoviruses, sapeloviruses and enteroviruses $B$ infecting domestic pigs and wild boars in the Czech Republic between 2005 and 2011. Infect Genet Evol. 2012;12:1447-51.

16. Wang C, Lan D, Hua X. Porcine kobuvirus from pig stool specimens in Shanghai, China. Virus Genes. 2011;43:350-2.

17. Bridger JC. Detection by electron microscopy of caliciviruses, astroviruses and rotavirus-like particles in the faeces of piglets with diarrhoea. Vet Rec. 1980;107:532-3.

18. Kroneman A, Cornelissen LA, Horzinek MC, de Groot RJ, Egberink HF. Identification and characterization of a porcine torovirus. J Virol. 1998;72:3507-11.

19. Feng L, Shi HY, Liu SW, Wu BP, Chen JF, Sun DB, Tong YE, Fu MS, Wang YF, Tong GZ. Isolation and molecular characterization of a porcine teschovirus 1 isolate from China. Acta Virol. 2007;51:7-11.

20. Di Bartolo I, Tofani S, Angeloni G, Ponterio E, Ostanello F, Ruggeri FM. Detection and characterization of porcine caliciviruses in Italy. Arch Virol. 2014;159:2479-84.

21. Meng L, Tao J, Li B, Ma Y, Cheng J, Zhang C, Liu H. [Clinical detection of seven porcine diarrheaassociated viruses and evolution analysis of porcine kobuvirus]. Sheng Wu Gong Cheng Xue Bao. 2017;33:1292-303. 
22. Wang E, Guo D, Li C, Wei S, Wang Z, Liu Q, Zhang B, Kong F, Feng L, Sun D. Molecular Characterization of the ORF3 and S1 Genes of Porcine Epidemic Diarrhea Virus Non S-INDEL Strains in Seven Regions of China, 2015. PLoS One. 2016;11:e0160561.

23. Zhang B, Tang C, Yue H, Ren Y, Song Z. Viral metagenomics analysis demonstrates the diversity of viral flora in piglet diarrhoeic faeces in China. J Gen Virol. 2014;95:1603-11.

24. Chen Q, Wang L, Zheng Y, Zhang J, Guo B, Yoon KJ, Gauger PC, Harmon KM, Main RG, Li G. Metagenomic analysis of the RNA fraction of the fecal virome indicates high diversity in pigs infected by porcine endemic diarrhea virus in the United States. Virol J. 2018;15:95.

25. Reuter G, Boldizsar A, Kiss I, Pankovics P. Candidate new species of Kobuvirus in porcine hosts. Emerg Infect Dis. 2008;14:1968-70.

26. Yu JM, Jin M, Zhang Q, Li HY, Li DD, Xu ZQ, Li JS, Cui SX, Yang SH, Liu N, Duan ZJ. Candidate porcine Kobuvirus, China. Emerg Infect Dis. 2009;15:823-5.

27. Khamrin P, Maneekarn N, Kongkaew A, Kongkaew S, Okitsu S, Ushijima H. Porcine kobuvirus in piglets, Thailand. Emerg Infect Dis. 2009;15:2075-6.

28. Park SJ, Kim HK, Moon HJ, Song DS, Rho SM, Han JY, Nguyen VG, Park BK. Molecular detection of porcine kobuviruses in pigs in Korea and their association with diarrhea. Arch Virol. 2010;155:180311.

29. Khamrin P, Maneekarn N, Hidaka S, Kishikawa S, Ushijima K, Okitsu S, Ushijima H. Molecular detection of kobuvirus sequences in stool samples collected from healthy pigs in Japan. Infect Genet Evol. 2010;10:950-4.

30. Barry AF, Ribeiro J, Alfieri AF, van der Poel WH, Alfieri AA. First detection of kobuvirus in farm animals in Brazil and the Netherlands. Infect Genet Evol. 2011;11:1811-4.

31. Reuter G, Boros A, Pankovics P. Kobuviruses - a comprehensive review. Rev Med Virol. 2011;21:3241.

32. Nantel-Fortier N, Lachapelle V, Letellier A, L'Homme Y, Brassard J. Kobuvirus shedding dynamics in a swine production system and their association with diarrhea. Vet Microbiol. 2019;235:319-26.

33. Xiong NN, Tao J, Zhang QZh, Zhang CL, Li BQ, Chang Hsh, Liu HL. Establishment and application of multiplex PCR for detection of four porcine viral diarrhea pathogens. Zhongguo Shou Yi Ke Xue (In Chinese). 2016;46(08):972-8.

\section{Figures}


Figure 1

A

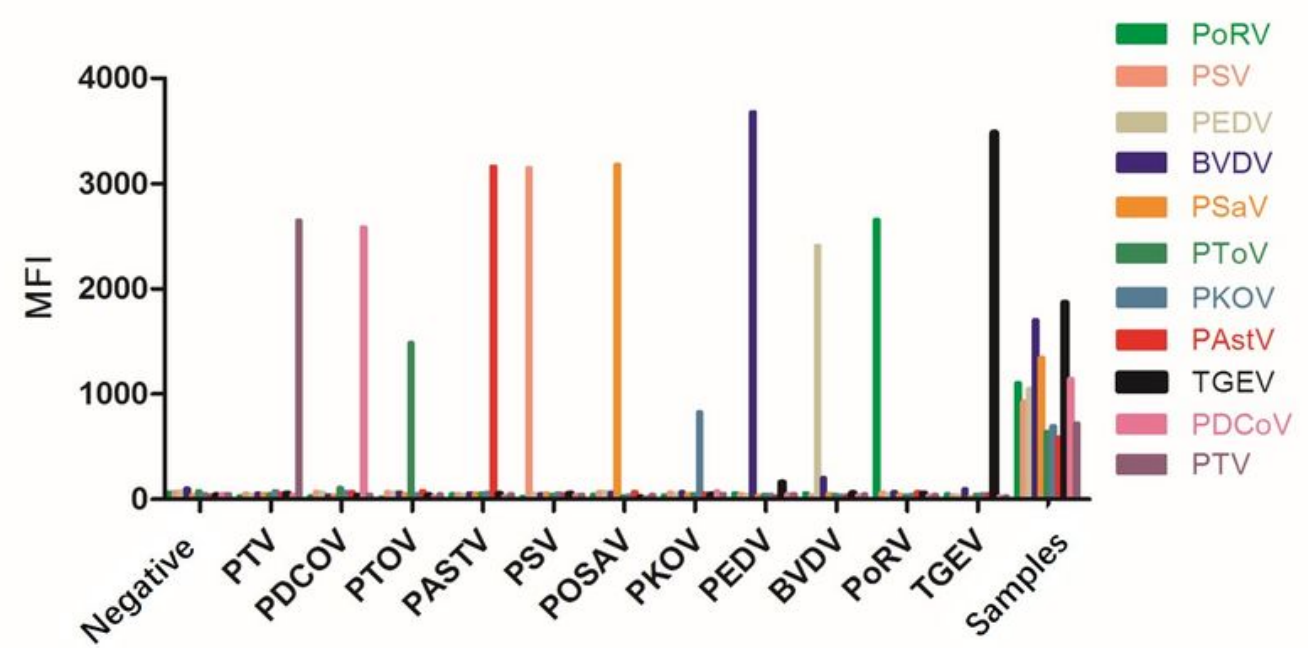

$\mathrm{B}$
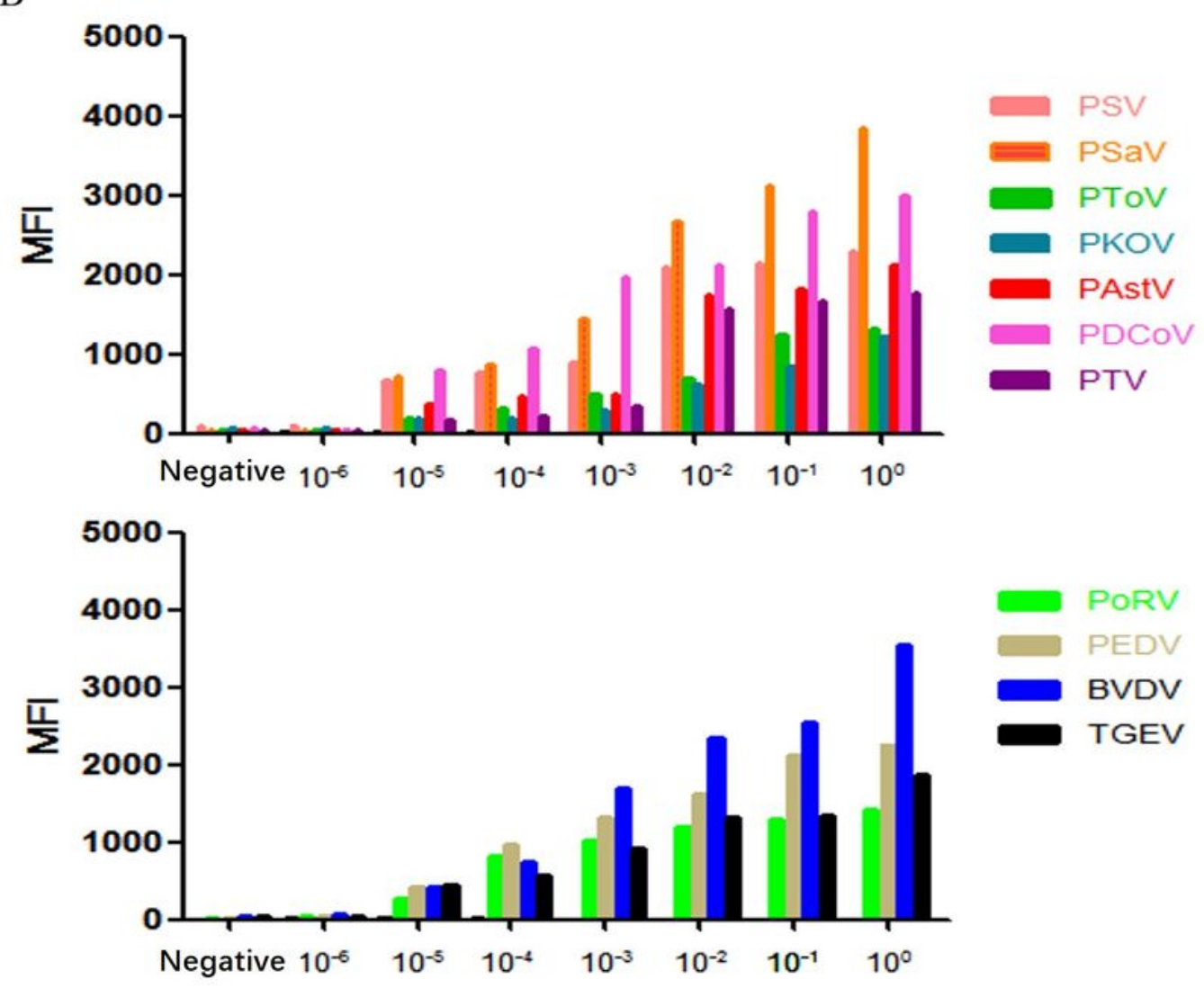

Figure 1

The specificity and sensitivity detection of the Luminex test. A. The specificity detection. B. The sensitivity detection. 
Figure 2

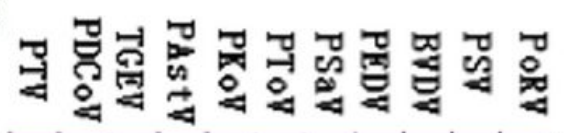

Hegetive

Sample24

Sample25

Sample26

Samp1e27

Samp1e28

Samp1e29

Sample30

Sample31

Sample 32

Sample33

Sample34

Sample35

Sample36

Sample37

Sample 38

Sample39

Sample40

Sample41

Samp1e42

Sample43

Sample44

Sample45

Sample46

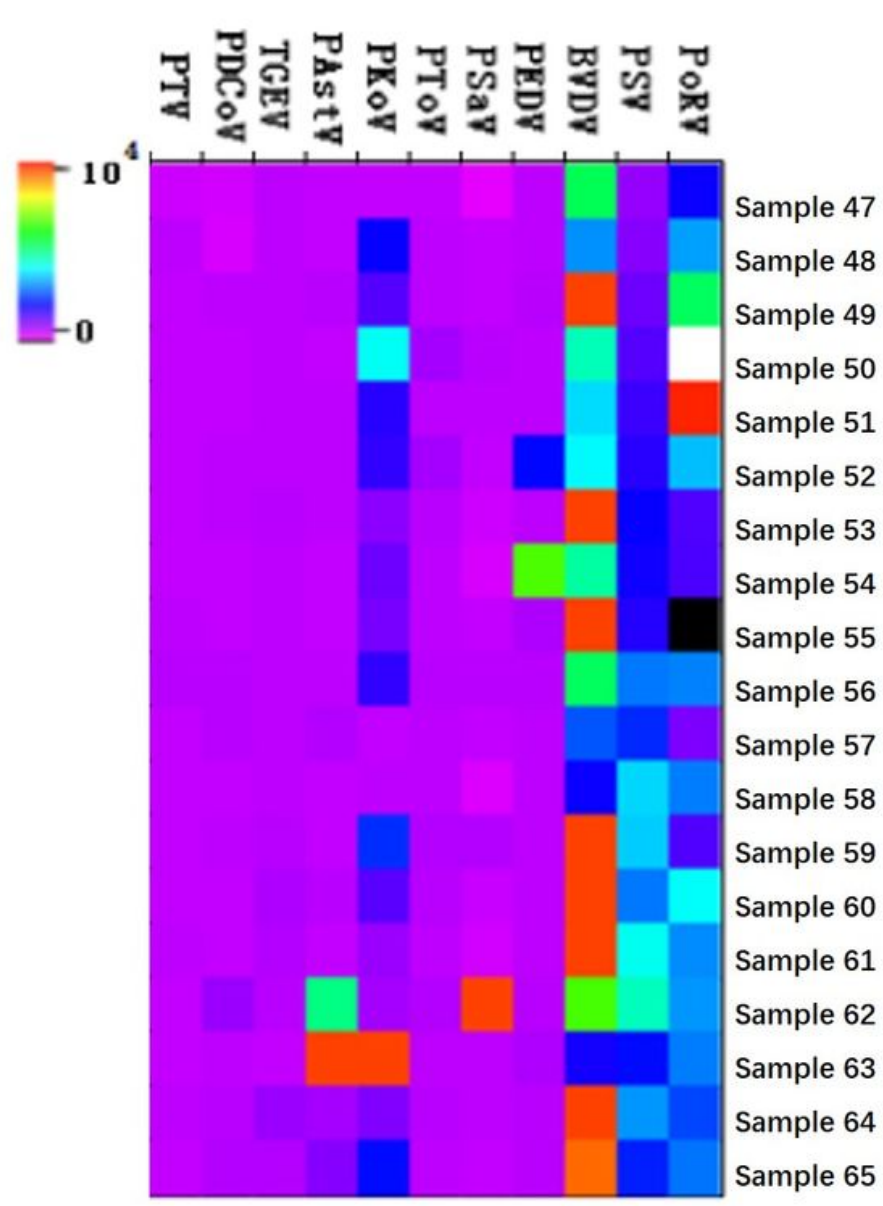

\section{Figure 2}

The Heatmap for quantitative results of some samples. The porcine stool specimens were tested.by the Luminex xTAG multiplex detection method. Only part data were present in the figure. 
Figure 3

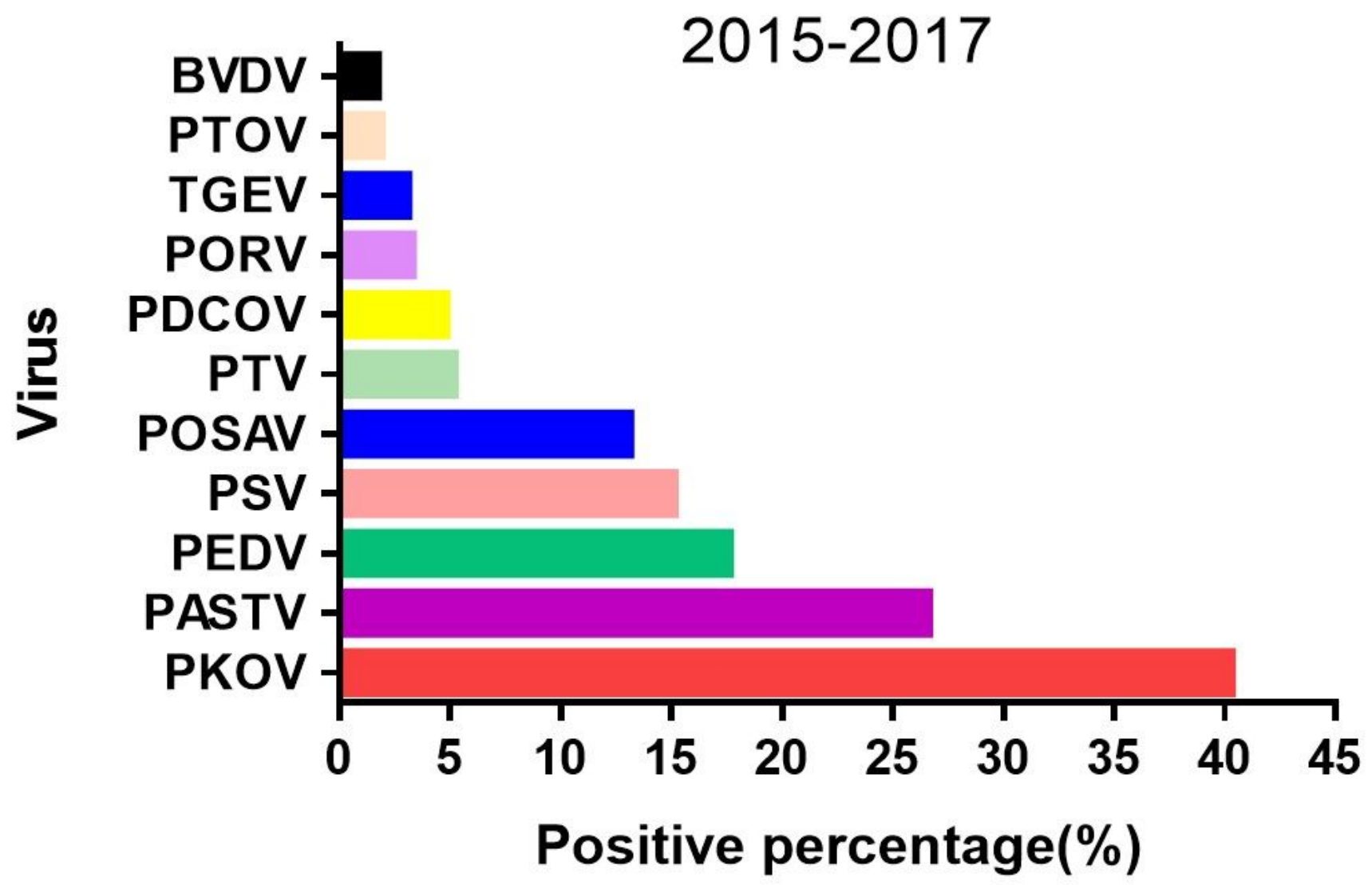

Figure 3

The positive percent of different enteric viruses from 2015 to 2017 in Shanghai. A total of 518 porcine stool specimens from 5 districts in Shanghai were detected by multiplex RT-PCR method. 
Figure 4

A

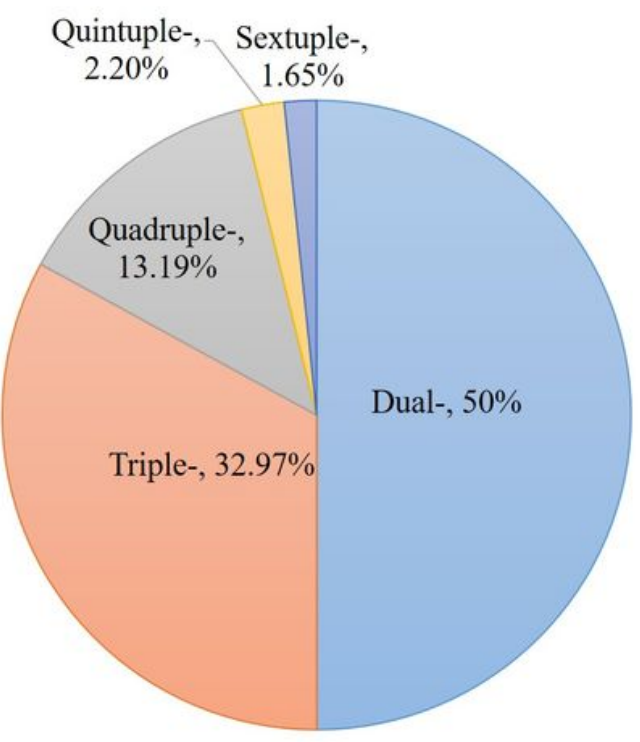

B

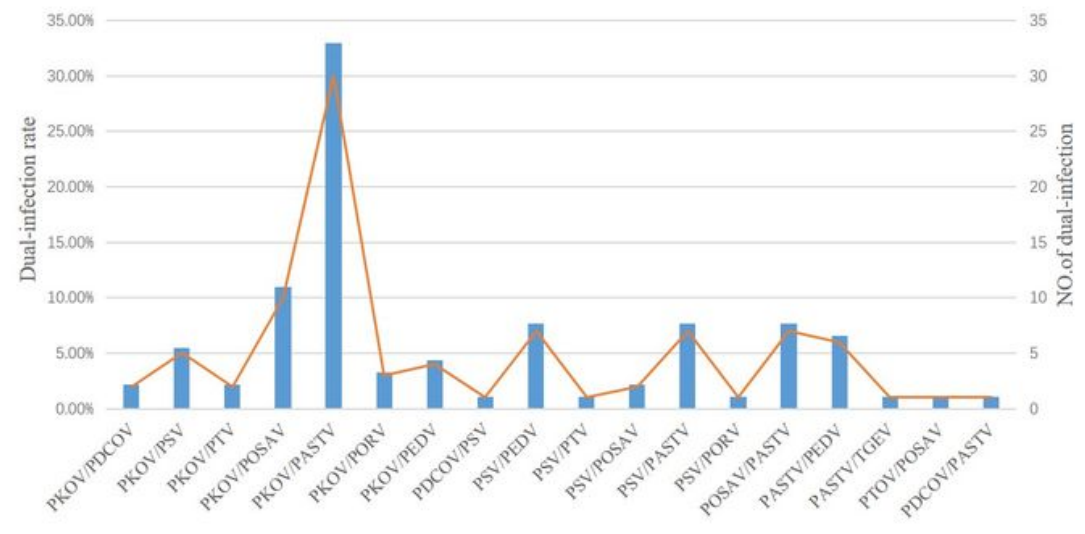

C
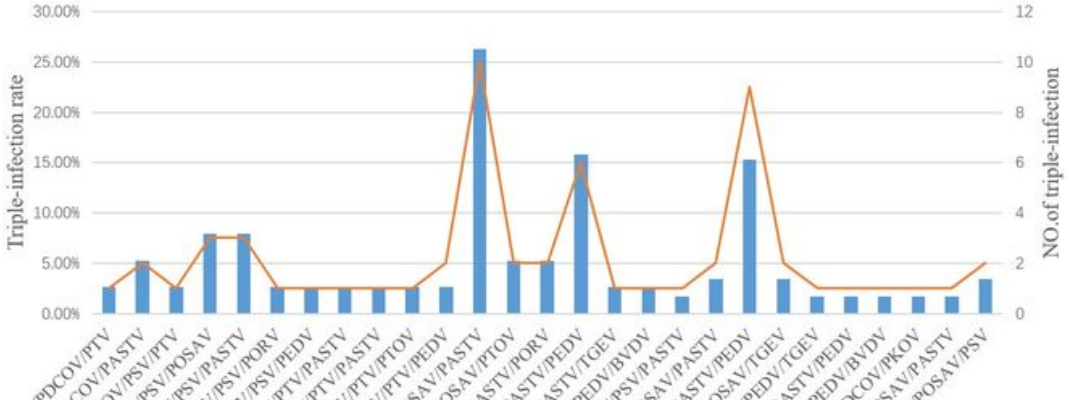

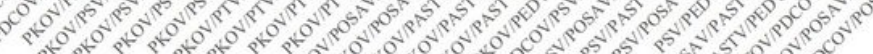

\section{Figure 4}

The multiple infections of different enteric viruses. Various combinations of co-infections were identified. A. The percent of multiple infections of different enteric viruses. B. The positive percent (left $Y$ axis) and positive numbers of dual-infections (right $Y$ axis) $C$. The positive percent (left $Y$ axis) and positive numbers of triple-infections. 
Figure 5

Farm A

\begin{tabular}{cccccccccccc}
\hline Virus & PKOV & PASTV & PSV & POSAV & PEDV & PORV & TGEV & PTOV & PTV & PDCOV & BVDV \\
\hline $\begin{array}{l}\text { Positive } \\
\text { percent }\end{array}$ & $68.67 \%$ & $32 \%$ & $24 \%$ & $24 \%$ & $12 \%$ & $11.33 \%$ & $8 \%$ & $2.67 \%$ & $1.33 \%$ & $1.33 \%$ & $0.67 \%$ \\
\hline
\end{tabular}

Farm B

\begin{tabular}{ccccccccc}
\hline Virus & PEDV & PSV & PKOV & PTV & PDCOV & PASTV & BVDV & PTOV \\
\hline $\begin{array}{c}\text { Positive } \\
\text { percent }\end{array}$ & $37.74 \%$ & $24.53 \%$ & $20.75 \%$ & $16.98 \%$ & $7.55 \%$ & $7.55 \%$ & $3.77 \%$ & $1.89 \%$ \\
\hline
\end{tabular}

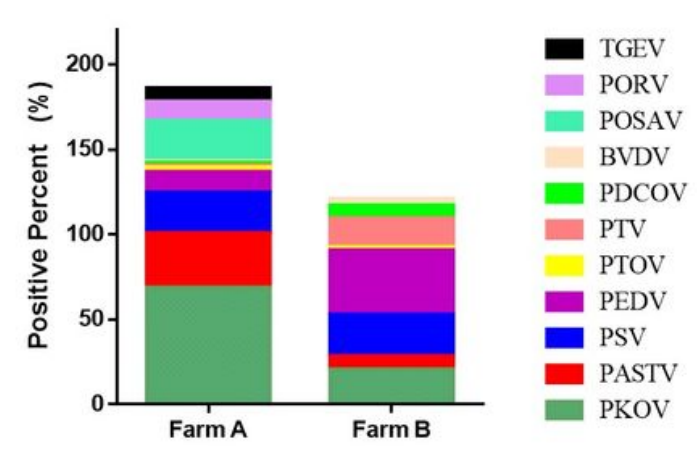

\section{Figure 5}

Detection of enteric viruses in different farms. The composition of enteric pathogens among different farms was analyzed. 
Figure 6

\begin{tabular}{|c|c|c|c|c|c|}
\hline & Dual- & Triple- & quadruple- & quintuple- & sextuple- \\
\hline Farm A & $20.67 \%$ & $19.33 \%$ & $8.67 \%$ & $0.67 \%$ & $0.67 \%$ \\
\hline Farm B & $26.42 \%$ & $5.66 \%$ & - & - & - \\
\hline
\end{tabular}

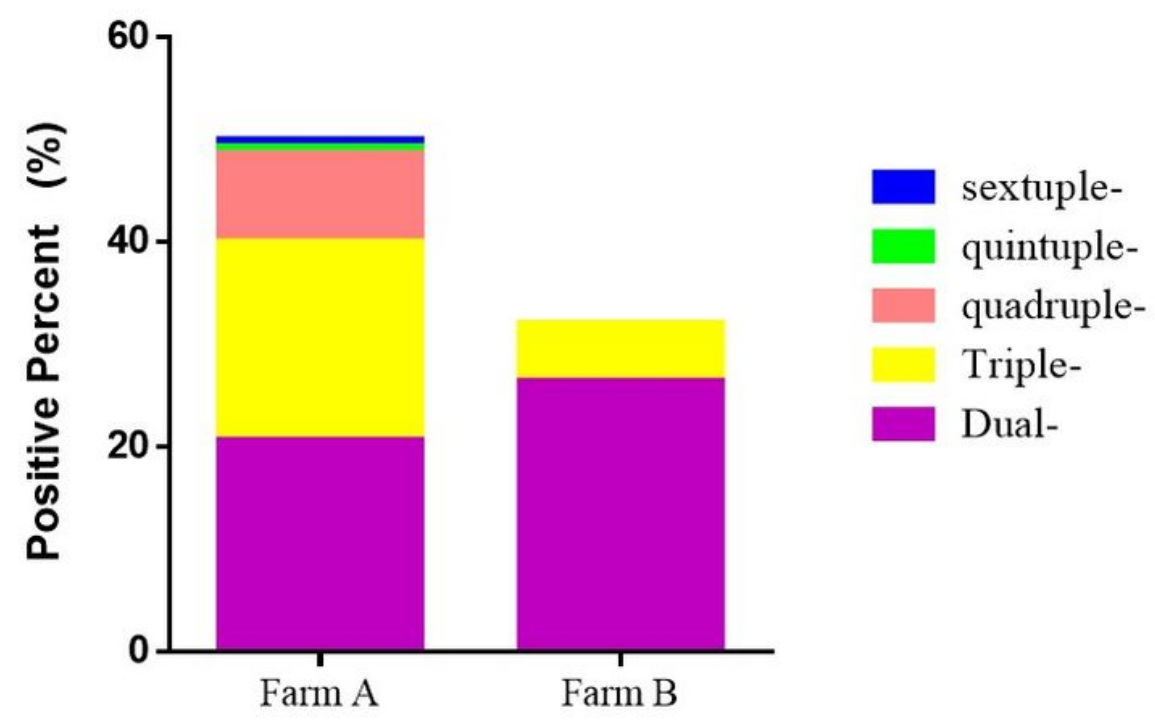

Figure 6

The co-infections of different farms. Different co-infections were analyzed in different farms. 
Figure 7

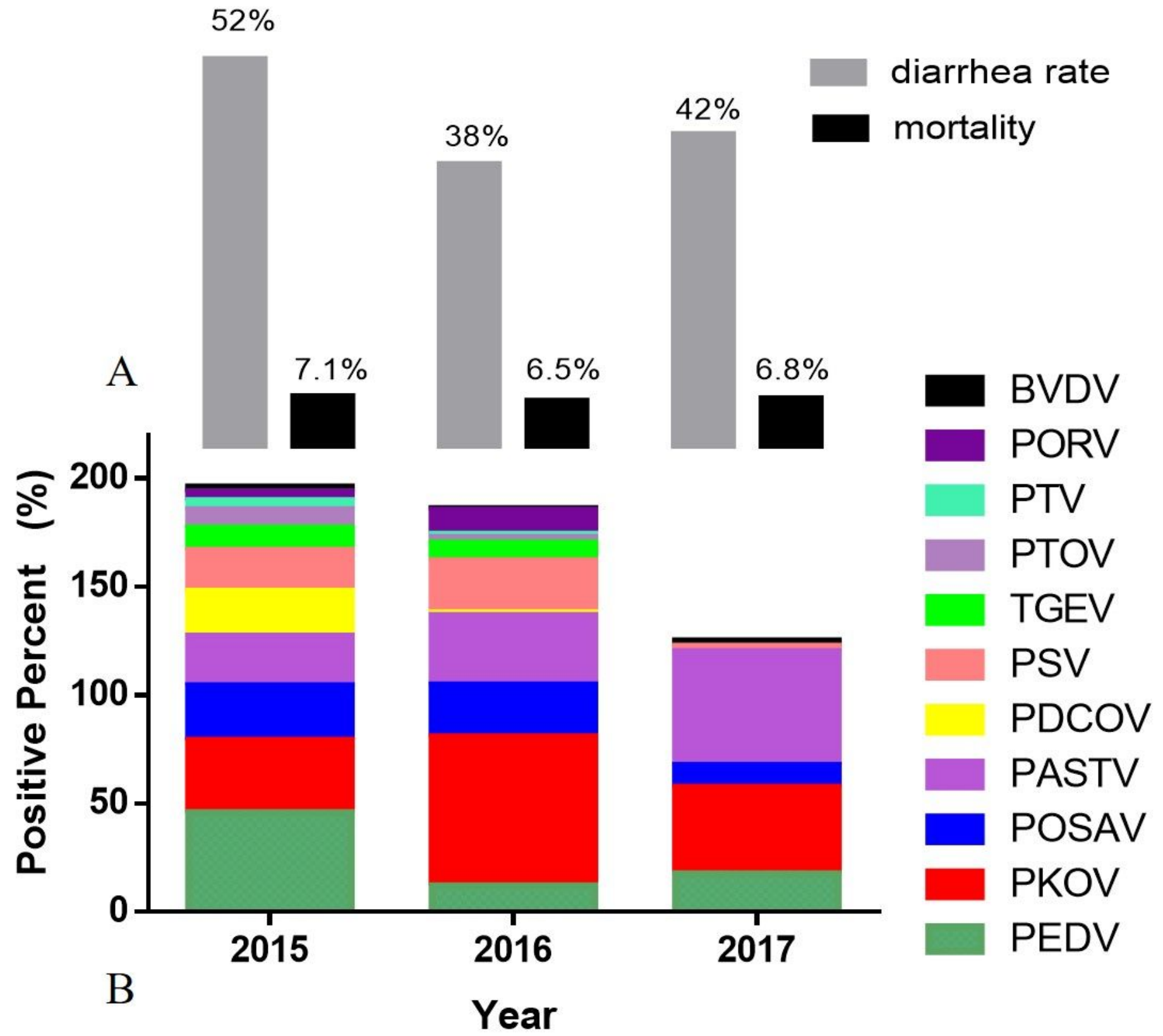

Figure 7

Pathogen spectrum of a specific farm from 2015 to 2017. The porcine stool specimens were detected by multiplex RT-PCR method every year from 2015 to 2017. It was observed that the prevalence of the viral diarrhea pathogens also changed over time. A. The diarrhea rate and mortality. B. The pathogen composition in different years. 